\title{
Nonlinear Identification of Glucose Absorption Related to Diabetes Mellitus
}

\author{
György Eigner*, Katalin Koppány ${ }^{\dagger}$, Péter Pausits ${ }^{\ddagger}$, Levente Kovács* \\ *Physiological Controls Research Center and Research, Innovation, and Service Center, \\ Óbuda University, Budapest, Hungary, \\ Email: \{eigner.gyorgy,kovacs.levente\}@ nik.uni-obuda.hu \\ ${ }^{\dagger}$ Budapest University of Technology and Economics, \\ Faculty of Electrical Engineering and Informatics, Budapest, Hungary \\ Email: kopkat93@gmail.com \\ $\ddagger$ Antal Bejczy Center for Intelligent Robotics and Research Center and Research, Innovation, and Service Center, \\ Óbuda University, Budapest, Hungary, \\ Email: peter.pausits@irob.uni-obuda.hu
}

\begin{abstract}
In case of biomedical researches we often have to deal with complicated biological phenomenons, which are usually described with complex mathematical models. In most cases these mathematical models and the systems to be modelled are also nonlinear. The appropriate adjustment of the parameters of these models is always a problem which is hard to be solved. To work with such complex models is essential in many research fields and application areas e.g. in personalized medicine or by the control of physiological processes. Although there are many identification techniques available, there is no general or "oven-ready" solution in cases where the mathematical model describing the dynamics of the physiological processes is highly nonlinear. One of our aims was to develop a simple, userfriendly and flexible identification framework which supports the identification of complex, nonlinear mathematical models. The performance of the method can be measured by simple metric. On the other hand, our goal was to successfully realize the identification framework in case of glucose absorption models, which are essential in our future work in order to validate the performance of advanced control algorithms. Our results show that the nonlinear identification framework performed well, since the predefined requirements were satisfied in all cases.

Index Terms-Nonlinear Least Mean Square-based Identification, Identification of Diabetes Mellitus, Glucose Absorption
\end{abstract}

\section{INTRODUCTION}

Diabetes mellitus is one of the most widespread diseases. There are more and more people affected by it every year. Hence the controlling of the blood glucose level is a major research area for engineers and doctors. Patients with type 1 diabetes mellitus (T1DM) can not control their blood sugar level, because their pancreas is unable to produce insulin, which is the key hormone of blood glucose regulation [1]. Therefore, these people need multiple daily insulin injections or they have to use an insulin pump. Insulin management is an energy- and time-consuming burden for the patients, but

Gy. Eigner was supported by the ÚNKP-17-4/IV. New National Excellence Program of the Ministry of Human Capacities. This project has received funding from the European Research Council (ERC) under the European Union's Horizon 2020 research and innovation programme (grant agreement No 679681) there is a special type of insulin pumps which can simplify this process and based on them the so-called artificial pancreas (AP) concept can be realized. The AP is a system combining a glucose sensor, a control algorithm, and an insulin infusion device to achieve the closed-loop control of the blood glucose level. Modern artificial pancreases use continuous glucose monitoring (CGM) technology. That means that the device measures glucose concentration - usually in the interstitium - via the glucose sensor every 5 minutes. Then, based on the measured values, the insulin infusion device injects a quantity of insulin which is required to keep the patient's blood sugar level in the normoglicemic range. So the AP literally copies the functioning of the human pancreas, and that way it can match the needs of every patient individually, even when they are exercising or eating different portions of meals. With suitable amounts of insulin the AP helps to prevent hyperglycaemia (high blood sugar concentration) and hypoglycaemia (low blood sugar concentration) with little or no input from the patient [2]-[6].

There exist many different glucose absorption models which could be applied and tested during the development of an artificial pancreas. Knowing and applying these absorption models are essential, since every control algorithm should be tested in in-silico trials before it can be used in clinical environment. However, the outputs of different models can not be compared, because in most cases it is not known, on what database the model parameters has been identified. To be able to compare their performances, the parameters have to be identified on the same database. This problem can be solved by using a general identification process which is able to adjust the selected parameters of the applied mathematical models. With this solution, a large population of virtual patients can be realized and the performance of any new advanced control algorithm in this regard can be validated in-silico before its validation in clinical environment. Accordingly, one of our goals was to develop an identification framework which is general and can be used regardless the physiological processes 
to be described and the mathematical model to be identified.

The paper is structured as follows: first, we introduce the applied glucose absorption models. Next, we present the developed identification framework. Then, we introduce the applicability of the identification framework in case of the introduced glucose absorption models. Finally, we submit our findings.

\section{Applied Glucose Absorption Models}

We applied two well-known glucose absorption models, the Hovorka model and the Magni model. The point of applying two different models was to be able to use one model to generate a data row and then adjust the parameters of the other model to fit that data row. This is the best approximation of testing the identification process on real patient data and in this way we can be sure that the performance of the framework is good enough to be used with real measured data, which are usually affected by several noises.

\section{A. The Hovorka Model}

The glucose absorption subsystem of the Hovorka model is a two compartment model which has one input, the meal disturbances, and it simulates the glucose absorption rate from the meals.

$$
D(t)=\frac{1000 \cdot(t)}{M_{w G}}
$$

where $d(t)$ is the meal input in $\mathrm{gCHO} / \mathrm{min}, D(t) \mathrm{mmol} / \mathrm{min}$ is the meal input and $M_{w G} \mathrm{~g} / \mathrm{mol}$ is the molecular weight of glucose. The Hovorka model describes the dynamic between the meal input and the states $D_{1}(t)$ and $D_{2}(t)$, both in mmol in the two compartments:

$$
\begin{gathered}
\dot{D}_{1}(t)=A_{G} D(t)-\frac{D_{1}(t)}{\tau_{D}} \\
\dot{D}_{2}(t)=\frac{D_{1}(t)}{\tau_{D}}-\frac{D_{2}(t)}{\tau_{D}}
\end{gathered}
$$

where $A_{G}$ is the carbohydrate bioavailability parameter and $\tau_{D}$ is a time constant which indicates how long it takes until the meal is digested and enters the blood stream.

The glucose absorption rate can be calculated as follows:

$$
U_{G}(t)=\frac{D_{2}(t)}{\tau_{D}}
$$

where $U_{G}(t) \mathrm{mmol} / \mathrm{min}$ is the glucose absorption rate.

\section{B. The Magni Model}

The input of the Magni model is also the consumed glucose, and the intestinal absorption of glucose is modelled by a threecompartment model:

$$
\begin{gathered}
Q_{s t o}(t)=Q_{\text {sto } 1}(t)+Q_{\text {sto } 2}(t) \\
\dot{Q}_{\text {sto } 1}(t)=-k_{\text {gri }} Q_{s t o 1}(t)+d(t) \\
\dot{Q}_{s t o 1}(t)=-k_{\text {gut }}\left(t, Q_{\text {sto }}\right) Q_{s t o 2}(t)+k_{\text {gri }} Q_{s t o 1}(t) \\
\dot{Q}_{g u t}(t)=-k_{\text {abs }} Q_{\text {gut }}(t)+k_{g} u t\left(t, Q_{\text {sto }}\right) Q_{s t o 2}(t)
\end{gathered}
$$

$$
R a(t)=\frac{f k_{a b s} Q_{g u t}(t)}{M_{w G}}
$$

where $Q_{\text {sto }} \mathrm{mg}$ gives the amount of glucose in the stomach ( $Q_{\text {sto } 1}$ is the solid phase and $Q_{s t o 2}$ is the liquid phase), $Q_{\text {gut }}$ $\mathrm{mg}$ is the amount of glucose in the intestine, $k_{\text {gri }} 1 / \mathrm{min}$ is the grinding rate, $k_{a b s} 1 / \mathrm{min}$ is the rate constant of intestinal absorption, $f$ is the proportion of the absorbed amount that actually appears in the plasma, $d \mathrm{mg} / \mathrm{min}$ is the consumed glucose, $R a \mathrm{mmol} / \mathrm{min}$ is the glucose rate of appearance in plasma, and $k_{\text {gut }}$ is the rate constant of gastric emptying, which is a nonlinear function of $Q_{s t o 2}$.

$$
\begin{aligned}
& k_{\text {gut }}\left(t, Q_{\text {sto }}\right)= k_{\text {min }}+\frac{k_{\text {max }}-k_{\text {min }}}{2}\left\{\operatorname { t a n h } \left[\alpha \left(Q_{\text {sto }}-\right.\right.\right. \\
&\left.-b \bar{D}(t))]-\tanh \left[\beta\left(Q_{\text {sto }}-a \bar{D}(t)\right)\right]+2\right\} \\
& \alpha= \frac{5}{2 \bar{D}(t)(1-b)}, \beta=\frac{5}{2 \bar{D}(t) a}, \\
& \bar{D}(t)=Q_{\text {sto }}(\bar{t})+\int_{\bar{t}}^{\bar{t}_{f}} d(\tau) \mathrm{d} t
\end{aligned}
$$

where $\bar{t}$ and $\bar{t}_{f}$ are the initial and final times of the last ingestion, and $a, b, k_{\max }$, and $k_{\min }$ are model parameters [7]

\section{The IDEnTIFICATION PRocess}

We decided to handle the parameter identification problem as a Non Linear Least Squares Optimization (NLSO) problem [8]. In this case the default cost function is the sum of squares of errors between the measured and simulated outputs. We have been realized the identification framework in the Mathworks' MATLAB system by using an embedded solver called lsqnonlin which is able to solve nonlinear least-squares data-fitting problems in the following form:

$$
\min _{\boldsymbol{\Omega}}\|f(\boldsymbol{\Omega})\|_{2}^{2}=\min _{\boldsymbol{\Omega}}\left(f_{1}(\boldsymbol{\Omega})^{2}+f_{2}(\boldsymbol{\Omega})^{2}+\ldots+f_{n}(\boldsymbol{\Omega})^{2}\right)
$$

where $f(\boldsymbol{\Omega})$ is the objective function to be minimized and $\Omega$ is the vector of the parameters to be identified. Rather than compute the sum of squares, the implemented lsqnonlin function requires a user-defined function to compute the real vector-valued function [9].

In order to handle the identification process appropriately we defined a difference based function which was applied in the following form:

$$
f_{1}(\boldsymbol{\Omega})=\sqrt{(\mathbf{z}-\hat{\mathbf{z}}(\boldsymbol{\Omega}))^{2}},
$$

at which the $\hat{\mathbf{z}}(\boldsymbol{\Omega})$ and $\mathbf{z}$ are the simulated data value and the target data value, respectively. From the investigated solutions (we examined different norm-based and absolute error functions as well) the (13) provided the best outcome in case of the given specific problem, namely, the smallest identification error based on the applied metric.

Therefore, we modified the (12) in accordance with the (13) in the following way:

$$
\min _{\boldsymbol{\Omega}}\|f(\boldsymbol{\Omega})\|_{2}^{2}=f_{1}(\boldsymbol{\Omega})^{2}
$$


The realized identification framework has many beneficial properties: it is easily adaptable to different nonlinear identification problems, cost effective and can be easily used. The model to be identified has to be realized in the SIMULINK environment - which is quite convenient solution -, however, the identification process is running under the MATLAB which makes the calling of external functions easy. However, these sub-systems cannot reach each other directly due to the specificities of the MATLAB system. In order to bypass this problem we have applied an intermediate layer through which the communication between the modules became possible. This layer is responsible for data synchronization and matching. The final structure of the framework can be seen on Fig. 1.

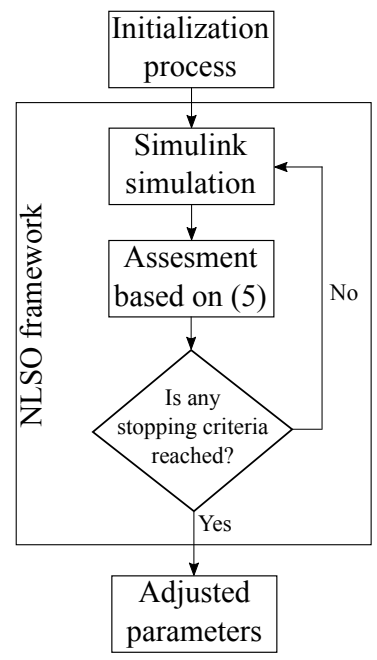

Figure 1. The core structure of the identification framework

As the Fig. 1. depicts, the identification framework can be divided into three parts: the core is the NLSO sub-system which is accompanied by the parameter initialization and adjustment sub-systems.

The first step of the identification process is the initialization of the sub-systems. The following parameters shall be determined preliminary:

- The external model parameters, namely the $\boldsymbol{\Omega}_{\text {init }}$.

- The parameters of the identification core. These are the internal and external parameters of the lsqnonlin solver and the determined boundaries of the parameters.

The followings need to be determined for the appropriate running of the algorithm: type and the termination (or stopping) criteria. The used algorithm type was the trust-region algorithm [10] which is numerically stable and robust, further, it allows the use of different freely defined cost functions. These were the applied termination criteria: $\mathbf{O}=[$ MaxIter,FunTol,StepTol,defaults $]=$ $[500,1 e-10,1 e-10,[]]$, where the MaxIter is the maximum number of allowed iterations, the FunTol is the termination tolerance on the function value, the StepTol is the termination tolerance on the maximum step distance and the [] means the not determined options which are defaults.

The $\boldsymbol{\Omega}_{u b}$ upper and $\boldsymbol{\Omega}_{l b}$ lower boundaries also needed to be set preliminary.

- The parameters of the SIMULINK simulator. These are the followings: the initial values of the SIMULINK model $\mathbf{x}(0)$, the sampling time $T$ - which shall be the same as the sampling time of the measurements $\mathbf{z}$ to keep the consistency -, the maximum step size of the simulation (we used 1 as maximum step size) and finally, the input related parameters (continuous or discrete).

The following steps summarize the operation of the NLSObased identification process:

1) Initialization. After the initialization the first $\hat{\mathbf{z}}(\boldsymbol{\Omega})$ appears through the simulation.

2) Investigation of the result. Based on the defined metric (5), the goodness of the $\hat{\mathbf{z}}(\boldsymbol{\Omega})$ is calculated.

3) Examination whether the algorithm reached the termination criteria in the given step or not. If one of the termination criteria is satisfied, then the iteration of the identification process stops. Otherwise, the iterations continue.

The outcome of the process is the adjusted $\Omega$ parameter set $\left(\boldsymbol{\Omega} \mid f_{1}(\boldsymbol{\Omega})^{2} \rightarrow \min \right)$.

\section{RESUlts}

During the investigation we applied simulated data instead of measured data - since one of the main purposes was to validate the operation of the identification framework. However, the simulated data coming from the virtual patient model was realistic and loaded by realistic noises.

We simulated the daily glucose intake of a man, so the simulation covers a period of $24 \mathrm{~h}$ and it includes five meals over the day. As a result of this simulation we get a vector with 1440 elements, because every minute has a corresponding value of glucose intake in $\mathrm{g} / \mathrm{min}$. In the next step we used the glucose intake vector as the input of one of the applied glucose absorption models to get a sample vector which contains the amounts of absorbed glucose in every five minutes in accordance with the capability of the CGM sensors. To test the performance of the identification algorithm we defined three scenarios as follows:

1) In the first scenario the model, which parameters were to be adjusted was the same as the model, which was used to generate the sample vector of the glucose absorption values in order to validate the prompt operation of the identification framework.

2) In the second scenario noise was added to the sample vector. In this case we had to consider the way of adding noise to the sample vector, because negative absorption values can physiologically not exist, so we had to avoid them. The noise was generated as a vector same size as the sample data vector and every element of the noise vector was a random number between -1 and 1 . 
Since the glucose absorption values are less than 20, these boundaries are suitable to generate noise which has an impact on the sample data, so we could test the identification algorithm in a more complex way than in scenario 1.

3) In the third scenario one model was used to generate the sample vector and then the algorithm adjusted the parameters of the other model to fit the sample vector. Noise was added to the sample vector in this case as well to simulate the measurement noise. This scenario is the closest approximation of adjusting the model parameters by using measurement data.

In case of both models we used zero initial values $\mathbf{x}\left(t_{0}\right)=$ $\mathbf{0}^{\top}$, namely, we considered that there has not been glucose intake before the simulated time duration. We used the same glucose intake vector in all three scenarios.

The effectiveness of the identification process was measured with a well-known quality metric called root mean square error (RMSE) [11]. We calculated it in vector form as follows:

$$
\operatorname{RMSE}=\sqrt{\frac{(\mathbf{z}-\hat{\mathbf{z}})^{\top}(\mathbf{z}-\hat{\mathbf{z}})}{N}},
$$

where $N$ is the number of samples of the measured data.

\section{A. Parameters of the Hovorka Model}

The following parameters of the Hovorka model can be adjusted by the identification algorithm: the carbohydrate bioavailability parameter $A g$ and the time constant which indicates how long it takes until the meal is digested and enters the blood stream $\tau_{D}$, so based on (12) the parameter vector to be adjusted is $\boldsymbol{\Omega}=\left[A g, \tau_{D}\right]^{\top}$. During the identification we considered the followings in terms of the applied lower and upper boundaries and the applied initial parameter set:

- The applied lower and upper boundaries were $\boldsymbol{\Omega}_{l b}=$ $[0.7,26]^{\top}$ and $\boldsymbol{\Omega}_{u b}=[1,55]^{\top}$ in accordance with [12] [13] and our previous studies.

- The initial parameter values were defined as random numbers between the preset lower and upper boundaries so we can run multiple tests and see if the change of the initial parameters has any influence on the results of the identification process.

\section{B. Parameters of the Magni Model}

The following parameters of the Magni model can be adjusted by the identification algorithm: the grinding rate $k_{g r i}$, the rate constant of intestinal absorption $k_{a b s}$ and the proportion of the absorbed amount that actually appears in the plasma $f$, so based on (12) the parameter vector to be adjusted is $\boldsymbol{\Omega}=\left[k_{\text {gri }}, k_{a b s}, f\right]^{\top}$. During the identification we considered the followings in terms of the applied lower and upper boundaries and the applied initial parameter set:

- The applied lower and upper boundaries were $\boldsymbol{\Omega}_{l b}=$ $[0.0279,0.0285,0.7]^{\top}$ and $\boldsymbol{\Omega}_{u b}=[0.0837,0.0855,1]^{\top}$, respectively. In the literature there are not any references on the boundaries of the parameters $k_{g r i}$ and $k_{a b s}$, so we had to define them. Since the mentioned parameters fall in the magnitude of $10^{-2}$, we defined the lower boundary as the $50 \%$ of the initial parameter value and the upper boundary as the $150 \%$ of the initial parameter value.

- The initial parameter values were defined as random numbers between the preset lower and upper boundaries - same as by the Hovorka model.

\section{Results of Scenario 1}

The results of identification concerning the first scenario can be seen on Figure 2. In the upper panel the blue line represents the absorption profile we wanted to approach by adjusting the parameters of the Hovorka model and the red dashed line represents the absorption profile generated using the parameters adjusted by the algorithm. The magenta line represents the absorption profile we wanted to approach by adjusting the parameters of the Magni model and the cyan dashed line represents the absorption profile generated by using the parameters adjusted by the algorithm. In the lower panel the green and black lines represent the error in both cases (green line - Hovorka model, black line Magni model), namely, the difference between the sample data and the result of the identification corresponding to each sample time. Despite of the applied 5 min sampling time in accordance with the capabilities of a usual CGM sensor - the plots in this paper contain continuous lines, based on linear approximation in order to increase the understandability of the results. Table I. provides an overview of the adjusted parameters, lower and upper boundaries and RMSE values. The identification algorithm performed perfectly in the first scenario, since the adjusted model parameters matched the parameters that we used to create the sample data every time we ran the identification algorithm. It can be seen on Figure 2. that the sample absorption profile and the profile generated using the adjusted parameters overlap. These results proved that the algorithm is functioning well and we can run more complex tests.

Table I

RESULTS OF THE IDENTIFICATION IN SCENARIO 1: INITIAL VALUES OF Model PARAmeters, LOWER AND UPPER Boundaries, AdJUSTED PARAMETERS AND RMSE VALUES - FIRST ROW: HOVORKA MODEL, SECOND Row: MAGNi MODEL

\begin{tabular}{|c|c|c|c|c|c|c|}
\hline & Par. & Init. & Lb. & Ub. & Ident. & RMSE \\
\hline \multirow{2}{*}{ Hov. } & $\mathrm{Ag}$ & 0.772 & 0.7 & 1 & 0.8 & \multirow{2}{*}{$5.400 \cdot 10^{-9}$} \\
\cline { 2 - 6 } & $\tau_{D}$ & 51.709 & 26 & 55 & 40 & \\
\hline \multirow{3}{*}{ Mag. } & $k_{\text {gri }}$ & 0.061 & 0.0279 & 0.0837 & 0.056 & \multirow{2}{*}{$5.011 \cdot 10^{-9}$} \\
\cline { 2 - 6 } & $k_{a b s}$ & 0.037 & 0.0285 & 0.0855 & 0.057 & \\
\cline { 2 - 6 } & $\mathrm{f}$ & 0.760 & 0.7 & 1 & 0.95 & \\
\hline
\end{tabular}

\section{Results of Scenario 2}

The results of testing the identification algorithm on noisy data can be seen on Figure 3. Same colors represent the same absorption profiles, identification results and errors as in the first scenario. As can be seen in Figure 3, the added noise did effect the sample data, but the RMSE values were good, taking 

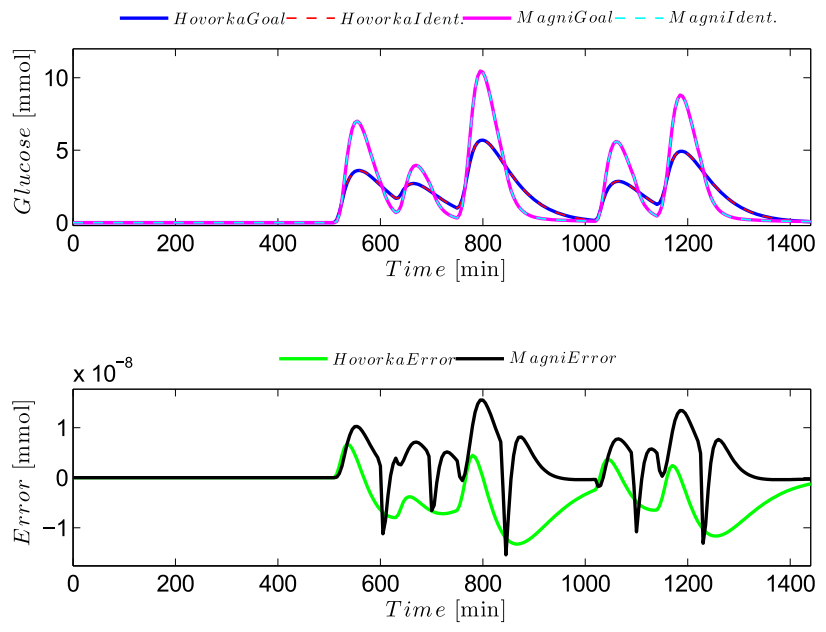

Figure 2. Results of the Identification in Scenario 1

into consideration the magnitudes of the values of the sample data. Table II. provides an overview of the adjusted parameters, lower and upper boundaries and RMSE values concerning the second scenario.
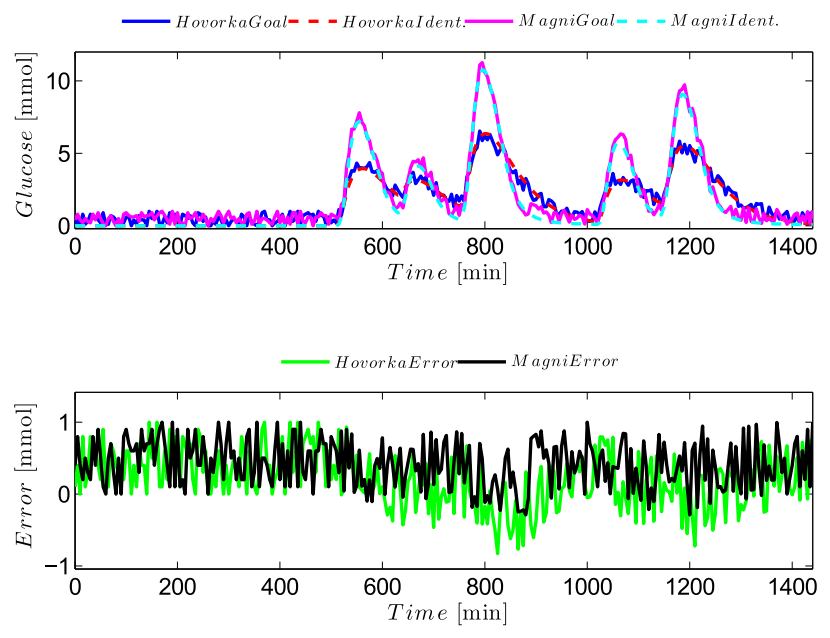

Figure 3. Results of the Identification in Scenario 2

\section{E. Results of Scenario 3}

This was the most realistic scenario which we set up to test the identification algorithm, namely, the sample data was generated with one absorption model, noise was added to it, and the algorithm adjusted the parameters of the other absorption model to fit the sample data. In the upper panel of Figure 4. the blue line represents the absorption profile of the Magni model which we wanted to approach by adjusting
Table II

RESUltS OF THE IDENTIFICATION IN SCENARIO 2: INITIAL VALUES OF Model PARAMETERS, LOWER AND UPPER BOUNDARIES, ADJUSTED PARAMETERS AND RMSE VALUES - FIRST Row: HovorKa MODEL, SECOND ROW: MAGNI MODEL

\begin{tabular}{|c|c|c|c|c|c|c|}
\hline & Par. & Init. & Lb. & Ub. & Ident. & RMSE \\
\hline \multirow{2}{*}{ Hov. } & $\mathrm{Ag}$ & 0.787 & 0.7 & 1 & 0.956 & \multirow{2}{*}{0.369} \\
\cline { 2 - 6 } & $\tau_{D}$ & 37.674 & 26 & 55 & 43.698 & \\
\hline \multirow{3}{*}{ Mag. } & $k_{\text {gri }}$ & 0.080 & 0.0279 & 0.0837 & 0.073 & \multirow{2}{*}{0.137} \\
\cline { 2 - 6 } & $k_{a b s}$ & 0.032 & 0.0285 & 0.0855 & 0.043 & \\
\cline { 2 - 6 } & $\mathrm{f}$ & 0.790 & 0.7 & 1 & 1 & \\
\hline
\end{tabular}

the parameters of the Hovorka model and the red dashed line represents the result of the identification. The magenta line represents the absorption profile of the Hovorka model which we wanted to approach by adjusting the parameters of the Magni model and the cyan dashed line represents the result of the identification. In the lower panel the green and black lines represent the error of the identification in each sample time, same color belongs to the same model as in the first and second scenarios. Table III. provides an overview of the adjusted parameters, lower and upper boundaries and RMSE values. The RMSE values were satisfying in this scenario as well.
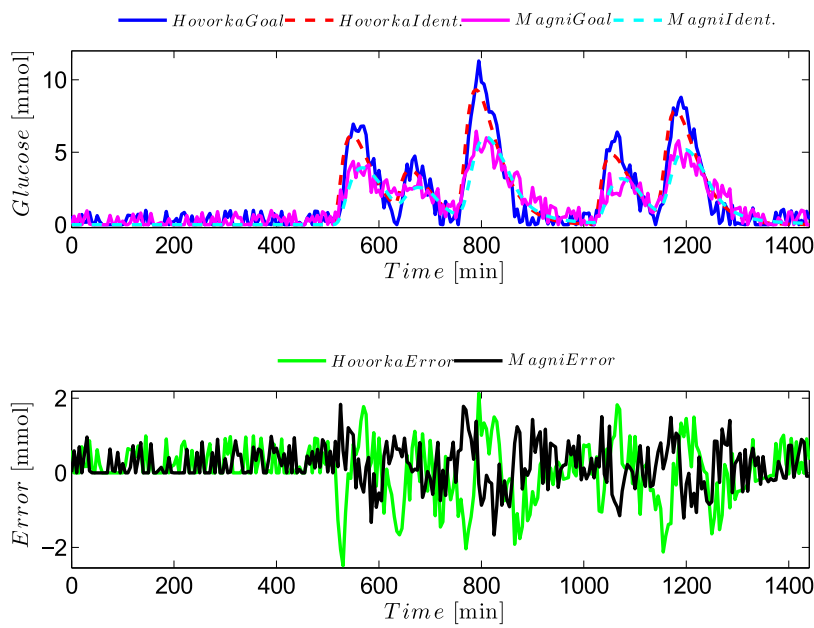

Figure 4. Results of the Identification in Scenario 3

\section{CONCLUSION}

In this paper we described a user-friendly and flexible nonlinear identification framework which can be used concerning to biomedical researches, e.g. in the field of physiological model identification. We systematically introduced the structure and the operation of the developed algorithm and the applied metric for the assessment. Our aim was to prove that the identification process is performing well in 
Table III

RESUlts OF THE IDENTIFICATION IN SCENARIO 3: INITIAL VALUES OF MOdEl PARAMETERS, LOWER AND UPPER BOUNDARIES, ADJUSTED PARAMETERS AND RMSE VALUES - FIRST Row: HovorKa MOdEL, SECOND Row: MAGNi MOdEL

\begin{tabular}{|c|c|c|c|c|c|c|}
\hline & Par. & Init. & Lb. & Ub. & Ident. & RMSE \\
\hline \multirow{3}{*}{ Hov. } & $\mathrm{Ag}$ & 0.814 & 0.7 & 1 & 1 & \multirow{2}{*}{0.759} \\
\cline { 2 - 6 } & $\tau_{D}$ & 82.850 & 26 & 55 & 28.873 & \\
\hline \multirow{3}{*}{ Mag. } & $k_{\text {gri }}$ & 0.069 & 0.0279 & 0.0837 & 0.029 & \multirow{2}{*}{0.600} \\
\cline { 2 - 6 } & $k_{a b s}$ & 0.071 & 0.0285 & 0.0855 & 0.043 & \\
\cline { 2 - 6 } & $\mathrm{f}$ & 0.984 & 0.7 & 1 & 0.754 & \\
\hline
\end{tabular}

in silico trial. To this end, we defined three scenarios with increasing complexity and we tested the algorithm using two well-known glucose absorption models. We applied the RMSE metric for the assessment of the operation of the identification framework. The identification algorithm performed well during our trials, even in the third scenario which was a close approximation of testing the algorithm on measured data. In our future work we will investigate the performance of the developed algorithm using measured patient data and we will apply the identification framework also on insulin absorption models.

\section{ACKNOWLEDGMENT}

The Authors thankfully acknowledge the support of the Robotics Special College of Óbuda University and the Óbuda University's Research, Innovation and Service Center.

\section{REFERENCES}

[1] A. Fonyó and E. Ligeti, Physiology (in Hungarian), 3rd ed. Budapest, Hungary: Medicina, 2008.

[2] M. Breton, A. Farret, D. Bruttomesso, S. Anderson, L. Magni, S. Patek, C. Dalla Man, J. Place, S. Demartini, S. Del Favero, C. Toffanin, C. Hughes-Karvetski, E. Dassau, H. Zisser, F. Doyle III, G. De Nicolao, A. Avogaro, C. Cobelli, E. Renard, and B. Kovatchev, "Fully integrated artificial pancreas in type 1 diabetes," Diabetes, vol. 61, pp. 2230-2237, 2011.

[3] C. Cobelli, E. Renard, and B. Kovatchev, "Artificial pancreas: Past, present, future," Diabetes, vol. 60, pp. 2672-2682, 2011.

[4] D. Drexler, J. Sápi, and L. Kovács, "Potential Benefits of Discrete-Time Controller-based Treatments over Protocol-based Cancer Therapies," ACTA Pol Hung, vol. 14, no. 1, pp. 11-23, 2017.

[5] Gy. Eigner, J.K. Tar, I. Rudas, and L. Kovacs, "LPV-based quality interpretations on modeling and control of diabetes," ACTA Pol Hung, vol. 13, no. 1, pp. 171-190, 2016.

[6] D. Copot, R. De Keyser, J. Juchem, and C. Ionescu, "Fractional Order Impedance Model to Estimate Glucose Concentration: in Vitro Analysis ," ACTA Pol Hung, vol. 14, no. 1, pp. 207-220, 2017.

[7] L. Magni, D.M. Raimondo, C. Dalla Man, G. De Nicolao, B. Kovatchev, and C. Cobelli, "Model predictive control of glucose concentration in subjects with type 1 diabetes: an in silico trial," in Proceedings of the 17th World Congress The International Federation of Automatic Control, 2008, pp. 4246-4251.

[8] T. Strutz, Data Fitting and Uncertainty (A practical introduction to weighted least squares and beyond), 2nd ed. Heidelberg, Germany: Springer Verlag, 2016.

[9] Mathworks Inc., Optimization Toolbox. Revised for Version 7.6 (Release 2017a), 2017. [Online]. Available: https://www.mathworks.com/help/ pdf_doc/optim/optim_tb.pdf

[10] T. Coleman and Y. Li, "Trust Region Approach for Nonlinear Minimization Subject to Bounds," SIAM J Optim, vol. 6, pp. 418-445, 1996.
[11] M. Tárnik, "T1DM model parameters adjustment based on PK, PD and CGM data," in INES 2015 - IEEE 19th International Conference on Intelligent Engineering Systems, 2015, pp. 313-318.

[12] R. Hovorka, F. Shojaee-Moradie, P.V. Carroll, L.J. Chassin, I.J. Gowrie, N.C. Jackson, R.S. Tudor, M. Umpleby, and D.H. Jones, "Partitioning glucose distribution/transport, disposal, and endogenous production during ivgtt," Am J Physiol Endocrinol Metab, vol. 282, no. 5, pp. E9921007, 2002.

[13] M. Naerum, "Model predictive control for insulin administration in people with type 1 diabetes," Technical University of Denmark, Tech. Rep., 2010. 